\title{
Improving the accuracy of free-form surface machining on CNC milling machines
}

\section{ANDRZEJ WERNER *}

Dr inż. Andrzej Werner, a.werner@pb.edu.pl,https://orcid.org/0000-0002-3768-5395 - Wydział Mechaniczny Politechniki Białostockiej, Białystok, Polska

This article presents a method of increasing the accuracy of the production of free-form surfaces. This method is based on the execution of coordinate measurements of the pre-treated object and reconstruction of its nominal geometric model in order to compensate existing machining errors. KEYWORDS: free-form surface, coordinate measurements, CNC milling machine, CAD model

\section{Introduction}

The proposed course of the process of manufacturing a surface object with machining deviation correction includes the following stages:

- construction of the nominal geometric model of the manufactured surface object,

- creation of machining programs,

- pre-treatment on a CNC milling machine,

- coordinate measurements of the object,

- determination of observed deviations and their components,

- determination of corrected coordinates at measuring points,

- reconstruction of the geometrical model of the object,

- creation of corrected machining programs and processing of the object,

- coordinate measurements and assessment of improving the accuracy of surface object manufacturing.

\section{Determination of corrected coordinates at measuring points}

Control coordinate measurements of a free surface panel with a rectangular contour can be carried out in the case of bidirectional, even distribution of measurement points. In this way, a grid of $n \times m$ observed points is obtained. For this purpose, automatic surface scanning procedures can be used, e.g. UVScan or Grid (PC-DMIS system). To estimate the manufacturing accuracy, machining deviations at the measuring points must be determined. The measure of deviations is the distance between points on the surface of the CAD model (nominal surface) and corresponding points observed during control measurements on the coordinate machine. Deviations are determined in a direction normal to the surface being machined (fig. 1).

Coordinate measurements provide information on the coordinates of nominal points, observed machining deviations and directional cosines at the measuring points. Based on this data, the surface patch describing the manufactured object can be corrected. First, the components of the observed machining deviations in the individual axes of the coordinate system are determined. The following relationships are used for the calculation:

$$
\begin{aligned}
& \Delta x_{i j}=d_{i j} \cdot \cos \alpha_{i j} \\
& \Delta y_{i j}=d_{i j} \cdot \cos \beta_{i j} \\
& \Delta z_{i j}=d_{i j} \cdot \cos \gamma_{i j}
\end{aligned}
$$

where: $\Delta x_{i j}, \Delta y_{i j}, \Delta z_{i j}$ - components of observed machining deviations; $d_{i j}$ - deviation observed at the measuring point; $\cos \alpha_{i j}, \cos \beta_{i j}$, $\cos \gamma_{i j}$ - directional cosines at measuring points; $i, j$ - coefficients describing location of the observed point. 


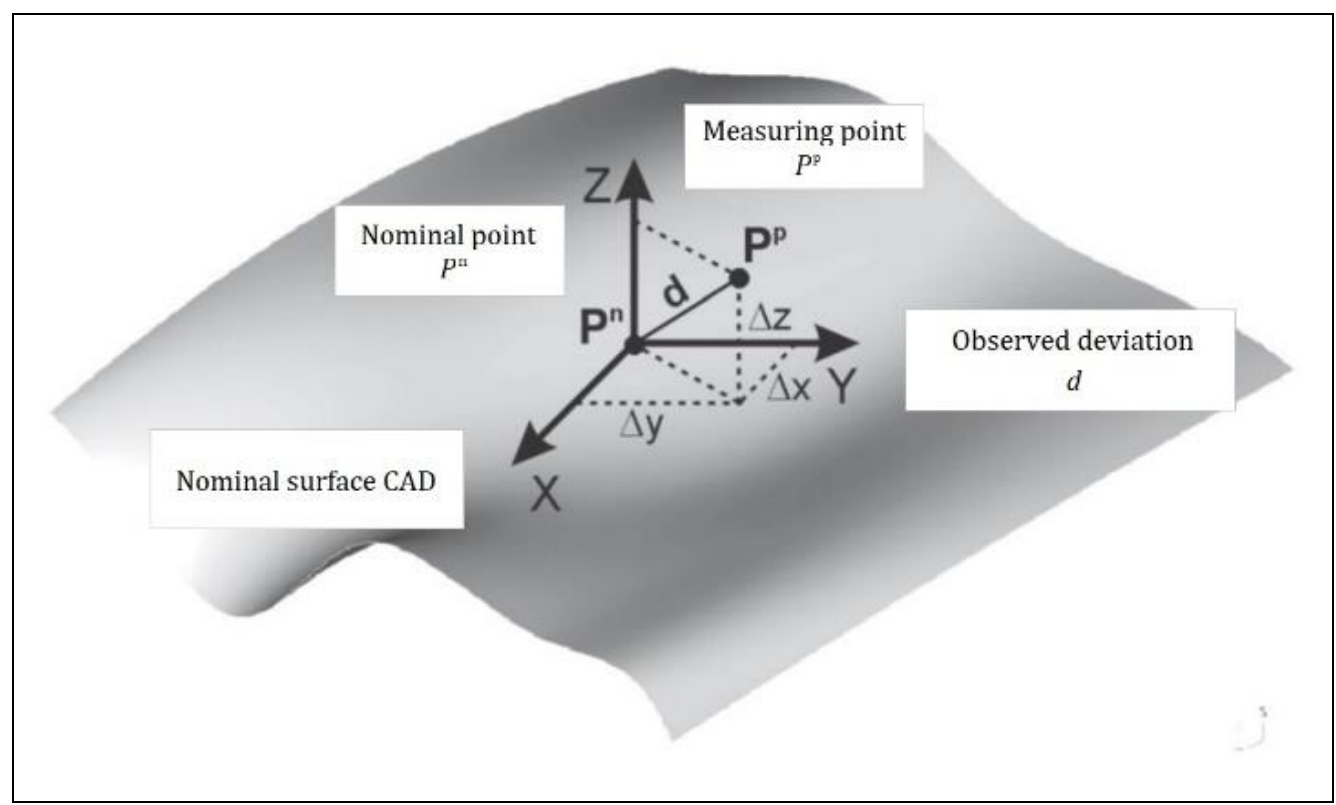

Fig. 1. Graphic representation of machining deviation

The determined components of machining deviations allow for calculation of corrected coordinates of points. In case the correction is carried out on raw measurement data, the corrected coordinates will be determined from the formula:

$$
\begin{aligned}
& x_{i j}^{\text {cor }}=x_{i j}^{\text {nom }}-\Delta x_{i j} \\
& y_{i j}^{\text {cor }}=y_{i j}^{\text {nom }}-\Delta y_{i j} \\
& z_{i j}^{\text {cor }}=z_{i j}^{\text {nom }}-\Delta z_{i j}
\end{aligned}
$$

where: $x_{i j}^{\text {cor }}, y_{i j}^{\text {cor }}, z_{i j}^{\text {cor }}-$ corrected coordinates of the surface panel; $; x_{i j}^{\text {nom }}, y_{i j}^{\text {nom }}, z_{i j}^{\text {nom }}-$ coordinates of points on the nominal surface (CAD model).

The approach presented is the simplest but does not guarantee achieving the best final result. Due to the complexity of the machining and measurement process, the observed deviations may contain significant effects of random phenomena. Deviations have two components: determined and random. Introduction of filtration of measurement data allows to minimize the impact of random deviations on the final effect of machining deviations correction. Formula (1) changes the form and components of corrected deviations are determined as follows:

$$
\begin{aligned}
\Delta x_{i j} & =d_{i j}^{\mathrm{f}} \cdot \cos \alpha_{i j} \\
\Delta y_{i j} & =d_{i j}^{\mathrm{f}} \cdot \cos \beta_{i j} \\
\Delta z_{i j} & =d_{i j}^{\mathrm{f}} \cdot \cos \gamma_{i j}
\end{aligned}
$$

where: $d_{i j}^{\mathrm{f}}$ - filtered components of observed machining deviations.

\section{Construction of the corrected surface panel}

The determined corrected coordinates are used to create a modified surface panel. Reverse engineering techniques are used in its construction. First, a grid of corrected $n \times m$ points is created (fig. 2a), and a series of curves is interpolated on it (fig. 2b), on which a lateral panel of surface (fig. 2c) is stretched. The surface panel constructed in this way compensates the machining deviations. It is necessary to modify machining programs. Method of correction of machining errors was verified on the example of the object described by means of the NURBS surface panel (fig. 3). It was built on a control grid consisting of 49 control points. The degree of B-glued basic functions in two directions of parameterization of the surface panel $u v$ was equal to 3 . The surface model was the basis for the preparation of programs controlling parts processing and measurement programs for checking the accuracy of execution. 


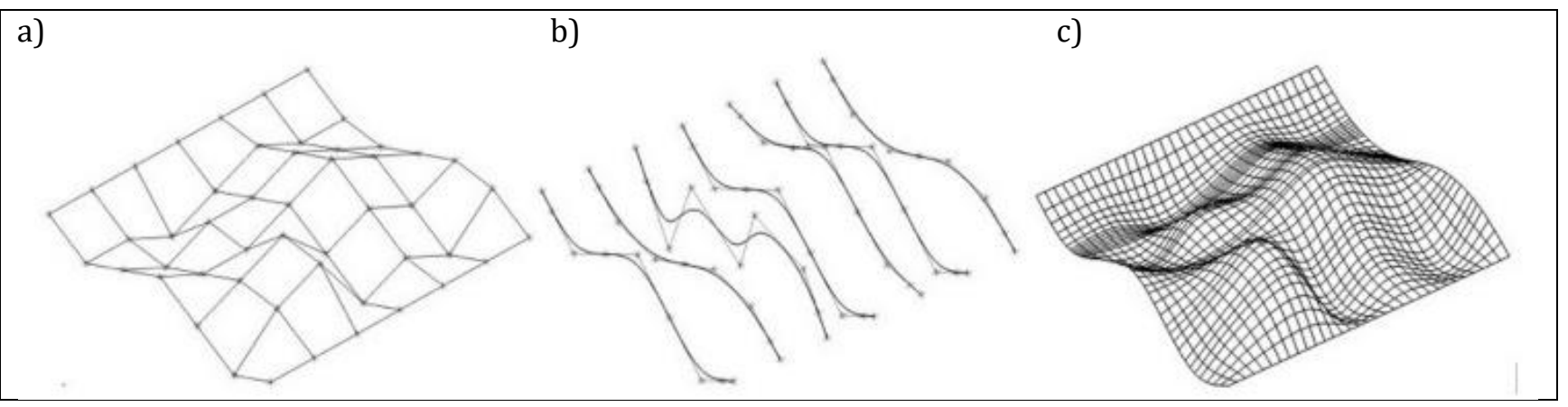

Fig. 2. Construction of the surface panel: a) grid of points, b) series of curves, c) surface panel

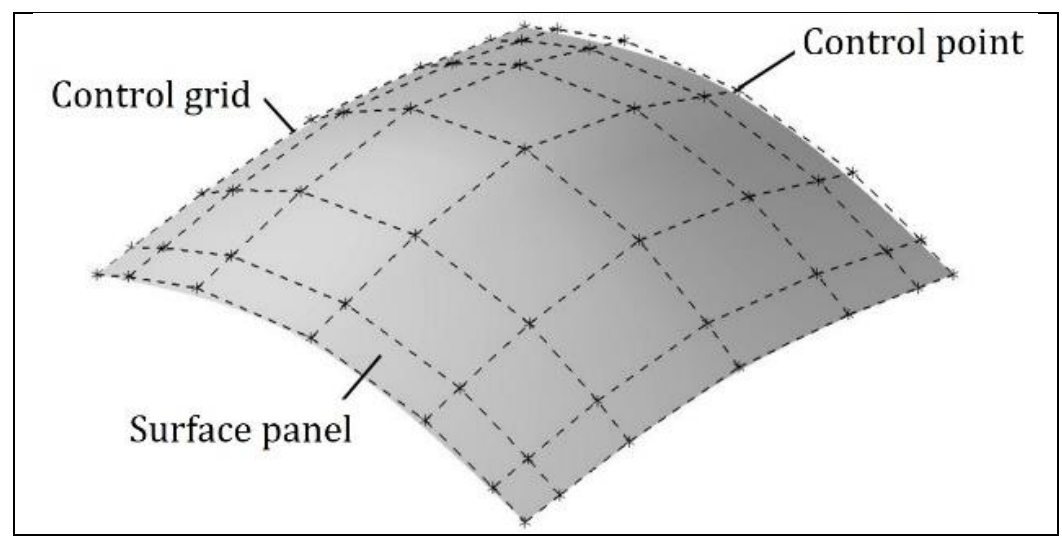

Fig. 3. Surface model describing the manufactured object

\section{Experimental verification of the proposed method to improve manufacturing accuracy}

The object was made of PA6 aluminum (fig. 4). After shaping, an allowance of $0.3 \mathrm{~mm}$ was left on the work surface. A $6 \mathrm{~mm}$ spherical cutter, designed for aluminum machining, was used to remove it. Parallel tool passes with $0.2 \mathrm{~mm}$ spacing have been programmed. Finishing was carried out at a spindle speed of $7500 \mathrm{rpm}$ and a feedrate of $300 \mathrm{~mm} / \mathrm{min}$.

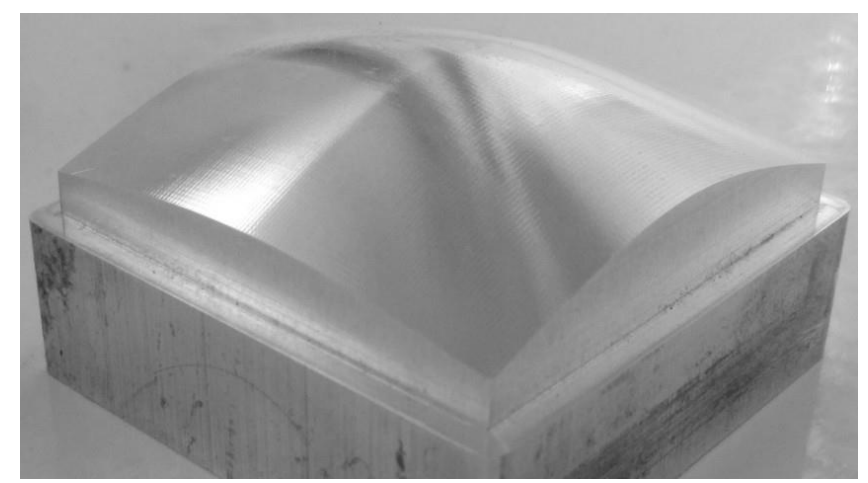

Fig. 4. The object created

After completing the manufacturing phase, the tested object was subjected to control measurements on a Global Performance measuring machine by Hexagon Metrology (PC-DMIS software, $M P E_{\mathrm{E}}=1.5+L / 333$ [ $\mu \mathrm{m}$ ], Renishaw SP25M measuring head, $20 \mathrm{~mm}$ long measuring probe with spherical tip with a diameter of $2 \mathrm{~mm}$ ). The measurements used the automatic UVscan surface scanning procedure, available in the PC-DMIS system. This procedure results in an even distribution of measuring points.

Finally, control measurements were programmed for a $45 \times 45$ grid of measuring points (distance between points $1 \mathrm{~mm}$ ). The distribution of measuring points on the measured surface is shown in fig. 5 .

The measurements provided information on 2025 observed deviations. The map of spatial deviations is presented in fig. 6 - all deviations were in the range $(-0,030 ;+0,045) \mathrm{mm}$. 


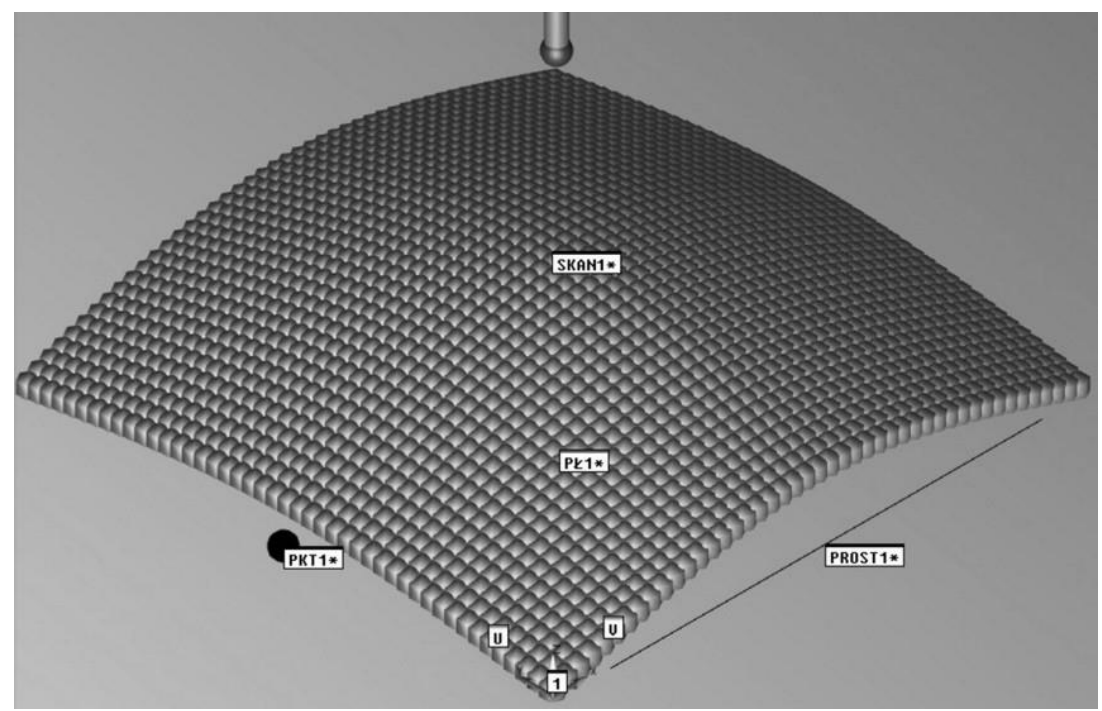

Fig. 5. Distribution of measuring points

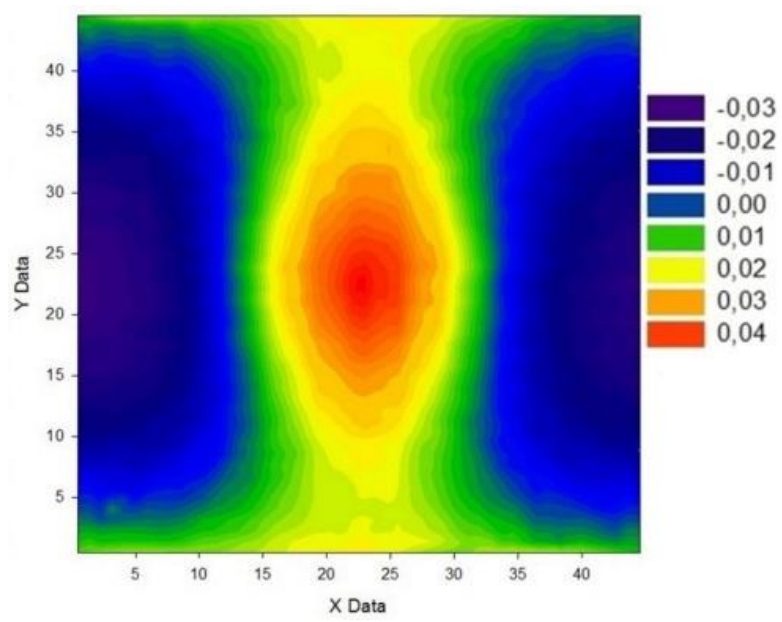

Fig. 6. Map of observed machining deviations

A summary of coordinate measurements of the machined surface is provided in Tab. I. The obtained accuracy was relatively good, and at the same time, it left a margin to apply the method of improving the final machining effects proposed in the paper.

TABLE I. Results of preliminary coordinate measurements - observed deviations

\begin{tabular}{|l|c|}
\hline Maximum negative deviation [mm] & -0.030 \\
\hline Maximum positive deviation [mm] & +0.045 \\
\hline Mean value [mm] & 0.0021 \\
\hline Standard deviation [mm] & 0.0173 \\
\hline
\end{tabular}

\section{Filtering of measurement data}

The paper proposes the adaptation of filters used for image processing [10]. The use of filters for processing the measurement data means that when calculating a new point value, point values from its surroundings are taken into account. Each measuring point in the environment has its own weight, which it brings into the calculations. These weights are saved as a mask. Typical mask sizes are: $3 \times 3,5 \times 5$ and $7 \times 7$. The principle of data filtration based on a filter with a $3 \times 3$ mask is as follows:

\begin{tabular}{|l|l|l|}
\hline$f_{-1,-1}$ & $f_{0,-1}$ & $f_{1,-1}$ \\
\hline$f_{-1,0}$ & $f_{0,0}$ & $f_{1,0}$ \\
\hline$f_{-1,1}$ & $f_{0,1}$ & $f_{1,1}$ \\
\hline
\end{tabular}


Deviations observed at the measuring points have the form of a grid consisting of $\mathrm{n}$ columns and $\mathrm{m}$ rows. The new value of the component of point $d_{i, j}$ with coordinates $(i, j)$ was calculated according to the following procedure. First, a weighted sum of the point component and all neighbours was determined, according to the weights indicated by the filter mask:

$$
\begin{gathered}
d_{i j}^{\prime}=f_{-1,-1} \cdot d_{i-1, j-1}+f_{0,-1} \cdot d_{i, j-1}+f_{1,-1} \cdot d_{i+1, j-1}+f_{-1,0} \cdot d_{i-1, j}+f_{0,0} \cdot d_{i, j}+ \\
+f_{1,0} \cdot d_{i+1, j}+f_{-1,1} \cdot d_{i-1, j+1}+f_{0,1} \cdot d_{i, j+1}+f_{1,1} \cdot d_{i+1, j+1}
\end{gathered}
$$

This result is divided by the sum of all mask weights (if it is different from 0):

$$
d_{i j}^{\mathrm{f}}=d^{\prime}{ }_{i j}:\left(f_{-1,-1}+f_{0,-1}+f_{1,-1}+f_{-1,0}+f_{0,0}++f_{1,0}+f_{-1,1}+f_{0,1}+f_{1,1}\right)
$$

By normalizing the component value of the observed machining deviation, a smoother distribution of the deviations is obtained and the impact of random components on the final result of the machining error correction is minimized. In order to test the procedure proposed in the article, a $5 \times 5$ mask was used. Due to the even distribution of measuring points, it was assumed that the influence of all points surrounding the processed deviation is equal (all weights are equal to 1).

The effect of data filtration, i.e. modified machining deviation map, is shown in fig. 7. In comparison with the raw deviation map (fig. 6), the outline lines representing individual levels of deviation were significantly smoothed. This is the result of minimizing the impact of random components generated during the machining and measuring process.

Table II contains numerical values that illustrate the change in raw deviations after applying data filtration. A change in the maximum values of deviations can be observed: the filtered components are in the range $(-0.002 ; 0.006)$, and their dispersion relative to the mean value (standard deviation) is insignificant.

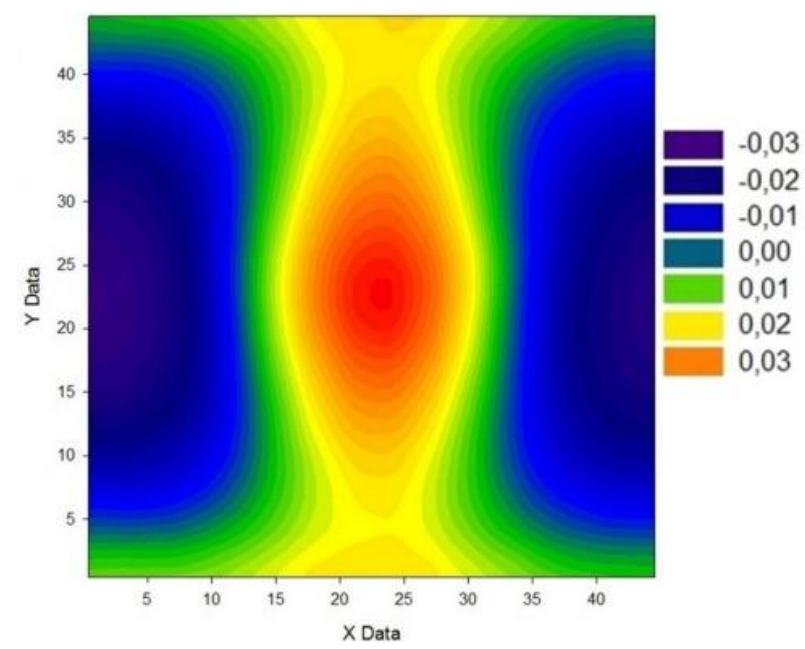

Fig. 7. Effect of filtration of observed machining deviations - modified deviation map

TABLE II. Filtration results of observed machining deviations

\begin{tabular}{|l|c|c|c|}
\cline { 2 - 4 } \multicolumn{1}{c|}{} & Raw deviations & Corrected deviations & $\begin{array}{c}\text { Filtered compo- } \\
\text { nents }\end{array}$ \\
\hline Maximum negative deviation [mm] & -0.030 & -0.028 & -0.002 \\
\hline Maximum positive deviation [mm] & 0.045 & 0.040 & 0.006 \\
\hline Mean value [mm] & 0.0021 & 0.0021 & $-2.1 \mathrm{E}-05$ \\
\hline Standard deviation [mm] & 0.0173 & 0.0168 & 0.0009 \\
\hline
\end{tabular}

\section{Improved accuracy of surface object production}

In accordance with the procedure previously described, construction of two corrected geometric models of the manufactured object was started. The first model was rebuilt based on raw coordinate measurement results, while in the second case, filtered machining deviations were used. At the initial stage of the correction 
process, nominal coordinates and observed coordinates of 2025 measuring points were separated from the measuring program. Using equations (1), (2) and (3), two sets of corrected coordinates (before and after filtration of measurement data) were determined. On their basis, two corrected geometric models of the produced surface were built in the MASTERCAM system. For each of them, a series of 45 interpolated curves were created on the grid of points. In the next phase, a panel of surface was stretched on the series of curves obtained.

Based on the corrected geometric models, machining programs were re-created. For this, the same tools and parameters were used as were adopted in the pre-treatment of the object. The re-created two surface panels were subjected to coordinate measurements. Maps of obtained machining deviations are shown in fig. 8. Table III, however, compares numerical values resulting from the correction of machining deviations. It should be noted that in both cases, significant improvement in manufacturing accuracy was achieved.

TABLE III. Summary of results of manufacturing accuracy improvement

\begin{tabular}{|l|c|c|c|}
\cline { 2 - 4 } \multicolumn{1}{c|}{} & Raw deviations & $\begin{array}{c}\text { Correction 1 - raw devi- } \\
\text { ations }\end{array}$ & $\begin{array}{c}\text { Correction 2 - filtered } \\
\text { deviations }\end{array}$ \\
\hline Maximum negative deviation [mm] & -0.030 & -0.005 & -0.004 \\
\hline Maximum positive deviation [mm] & 0.045 & 0.009 & 0.007 \\
\hline Mean value [mm] & 0.0022 & 0.0023 & 0.0013 \\
\hline Standard deviation [mm] & 0.0173 & 0.0018 & 0.0014 \\
\hline
\end{tabular}

a)

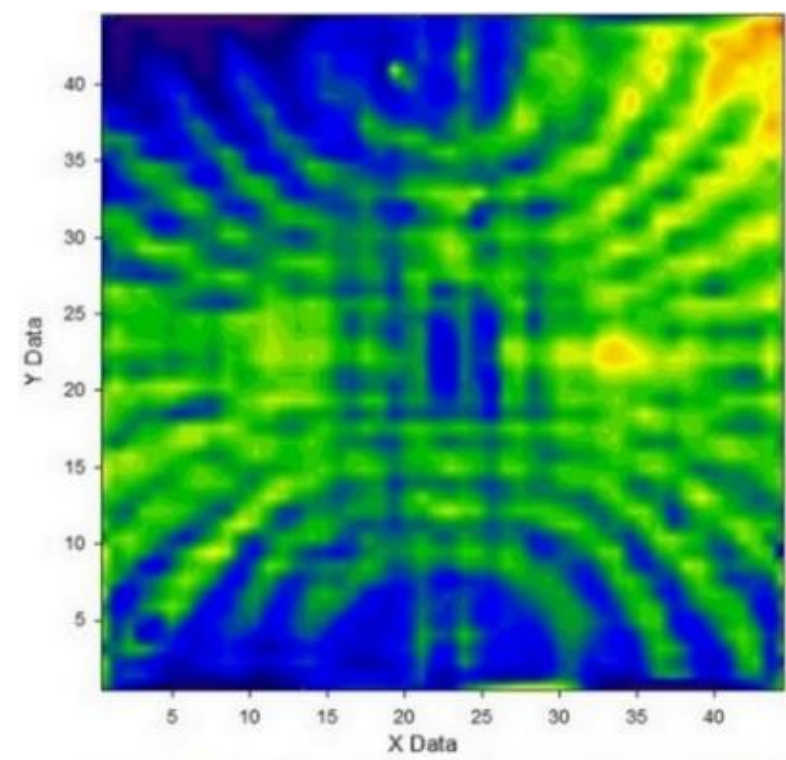

b)

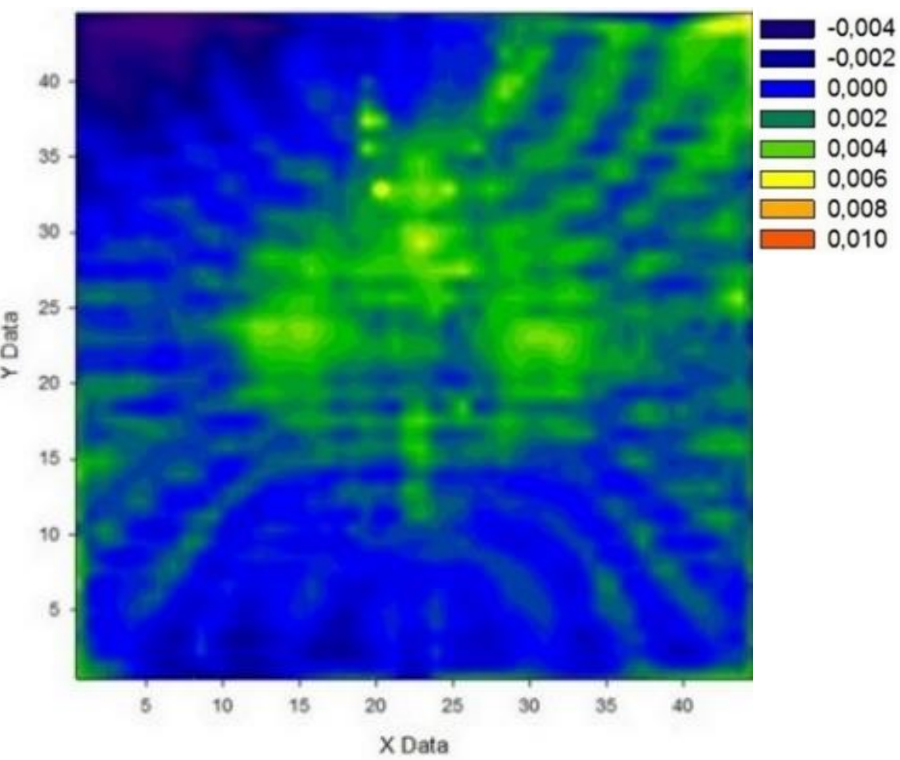

Fig. 8. Maps of deviations after correction of: a) raw measurement data, b) filtered measurement data

\section{Summary}

Application of the method presented in this paper resulted in a significant improvement in the accuracy of free surface production, which is confirmed by the results presented in tab. III. In both correction cases, there was a clear decrease in the maximum observed machining deviations. Filtration of measurement data improved the final result - the maximum machining deviations observed are the smallest in this case; also the standard deviation of the deviations and their mean indicate an additional positive effect. The created surface has the greatest similarity to the nominal CAD model. Filtration of measurement data allows to reduce the impact of random components of observed machining deviations on the process of improving the manufacturing accuracy.

Correction of manufacturing errors according to the presented method is relatively simple. It is based on typical equipment and software used in companies (CAD/CAM systems, CNC machine tools, coordinate measuring machines). Correction of machining errors is further facilitated by linking parametric technological and geometric data in modern CAD/CAM systems - technological data once processed in this way does not require re-entering into the system. As a result, after rebuilding the geometric model of the object, the tool path automatically rebuilds. 


\section{REFERENCES}

[1] Ramesh R., Mannan M.A., Poo A.N. "Error compensation in machine tools - a review. Part I: Geometric, cutting-force induced and fixturedependent errors". International Journal of Machine Tools \& Manufacture. 40 (2000): 1235-1256.

[2] Ramesh R., Mannan M.A., Poo A.N. "Error compensation in machine tools - a review. Part II: Thermal errors". International Journal of Machine Tools \& Manufacture. 40 (2000): 1257-1284.

[3] Zhengchun Du, Shujie Zhang, Maisheng Hong. "Development of a multi-step measuring method for motion accuracy of NC machine tools based on cross grid encoder". International Journal of Machine Tools \& Manufacture. 50 (2010): 270-280.

[4] Mehrdad V.N., Mohsen H., Behrooz A. "Tool path accuracy enhancement through geometrical error compensation". International Journal of Machine Tools \& Manufacture. 51 (2011): 471-482.

[5] Ryu S.H., Chu C.N. "The form error reduction in side wall machining using successive down and up milling". International Journal of Machine Tools \& Manufacture. 45 (2005): 1523-1530.

[6] Yang M.Y., Choi J.G. "A tool deflection compensation system for end milling accuracy improvement". Journal of Manufacturing Science and Engineering. 120 (1998): 222-229.

[7] Landon Y., Segonds S., Mousseigne M., Lagarrigue P. "Correction of milling tool paths by tool positioning defect compensation". Journal of Engineering Manufacture. Proceedings of the Institution of Mechanical Engineers. Part B. 217 (2003): 1063-1073.

[8] Cho M.W., Seo T.I., Kwon H.D. "Integrated error compensation method using OMM system for profile milling operations". Journal of Materials Processing Technology. 136 (2003): 88-99.

[9] Poniatowska M., Werner A. "Fitting spatial models of geometric deviations of free-form surfaces determined in coordinate measurements". Metrology and Measurement Systems 17, 4 (2010): 599-610.

[10] Kawasaki T., Jayaraman P.K., Shida K., Zheng J., Maekawa T. "An image processing approach to featurepreserving B-spline surface fairing". Computer-Aided Design. 99 (2018): 1-10. 